\title{
Einige Beobachtungen zur hochmittelalterlichen Geschichte der Komburg ${ }^{1}$ Vortrag auf der Komburg am 11. Juni 2016 anlässlich der Jahreshauptversammlung des Historischen Vereins für Württembergisch Franken
}

\author{
von ROLF SCHWEIZER ${ }^{2}$
}

Im Juni 1965 hat mich der damalige Leiter des Amtes für mittelalterliche Bodendenkmalpflege, Günther Fehring, auf die Komburg gerufen, um die Freilegung der wenige Tage zuvor von Eduard Krüger entdeckten Krypta unter dem Ostchor zu begleiten. Unsere Tätigkeiten waren an den Fortgang der Erneuerungsarbeiten des zuständigen Hochbauamtes gekoppelt, finanziell wie zeitlich, und deshalb auch öfters wochenlang unterbrochen. Diese Arbeit wurde mit der Ämterreform der 1970er Jahre beendet, und sie erstreckte sich schrittweise vom Ostchor der Kirche bis hin zum westlichen Kirchturm. Seither sind 50 Jahre vergangen und es gibt außer dem Vortragenden nur noch einen Zeitzeugen, welcher täglich dabei war, Herrn Bruno Schwenger. Die Komburg verbindet uns beide seit mehr als einem halben Jahrhundert. ${ }^{3}$

Vor wenigen Wochen war ich mit meinem Freund Gerhard Fritz im Gespräch unter anderem über die Komburg. Er meinte: „Solange du alles noch geordnet im Kopf hast, solltest du darüber öffentlich reden“. In der Tat könnte man noch

1 Im Folgenden wurde der Vortragscharakter des Textes grundsätzlich beibehalten, der Text allerdings durch einige Literaturhinweise ergänzt.

2 Zur allgemeinen mittelalterlichen Geschichte der Komburg: Rainer Jooß: Kloster Komburg im Mittelalter (FWFr 4). Sigmaringen ${ }^{2} 1987$ (z. T. ausführlicher: ${ }^{1} 1971$ ) sowie ders.: Bemerkungen zur Bau- und Patroziniengeschichte der Komburg. In: WFr 65 (1981), S. 109-112; Fritz Arens: Das Rätsel der Sechseckkapelle auf Großcomburg. In: ebd., S. 51-100; Hans Joachim von Brockhusen: Zum Ritterfresko in der Sechseckkapelle auf Groß-Komburg. In: ebd., S. 101-108; Horst Wengerter: Der romanische Kapitelsaal auf Großkomburg. Untersuchungen nach der ehemaligen Größe, Form und Ausstattung des 12. Jahrhunderts. In: ebd., S. 113-128; Hanna Hien: Das Kloster Komburg im Mittelalter. Monastisches Leben am Rande der fränkischen Klosterlandschaft. In: WFr 95 (2011), S. 7-24.

$3 \mathrm{Zu}$ den Ausgrabungen ist seinerzeit nur ein Vorbericht erschienen: Günter P. Fehring / Rolf Schweizer: Großkomburg. Der romanische Gründungsbau der Klosterkirche und seine Geschichte. In: WFr 56 (1972), S. 5-29. Noch einmal mit der Materie befasst hat sich Ulrike Plate: Ein Forschungsbericht zur Geschichte der Großkomburg. In: WFr 78 (1994), S. 7-36, vgl. das Bild von der Brunnenkapelle S. 35. 
vieles über die damalige Grabung erzählen. So habe ich beschlossen, heute eigentlich nur über den Stifter und die ersten 25 Jahre seiner Klostergründung zu sprechen.

\section{Der „Bebauungsplan“: Die Ideen des Grafen Burkhard und deren Verwirklichung von 1075 bis zu seinem Tod}

Seit der Untersuchung seiner sterblichen Reste (1948/50) wissen wir: Graf Burkhard lebte als Behinderter schwerkrank auf der Burg. Rückgratverkrümmungen, Gicht und Rheuma zeichneten seinen Körper. Diese Beschwernisse jedoch scheinen seinen Geist und sein Tun nicht beschränkt, eher beflügelt zu haben. Die Sorgen um das Seelenheil der ganzen Familie führten zur Idee, auf den Platz der stolzen Burg ein Kloster zu stellen. War dieser Mann ein weitsichtiger Denker und Ideengeber oder war er ein Phantast? Hatte er einen Stab fähigster Leute um sich geschart, um die Gedanken in Taten umzusetzen? Allein aus dem Baubestand der alten Klosteranlage, die uns weitgehend erhalten geblieben ist, lassen sich die kühnen, fast wahnwitzigen Ideen Burkhards erkennen, zugleich aber auch die technischen Fähigkeiten erahnen, was die Realisierung dieses Bebauungsplanes angeht.

Das neue Kloster soll auf dem von Ost nach West gerichteten, langgestreckt schmalen Felsgelände der Burg, und zwar in streng axialer Symmetrie errichtet werden. Dabei negiert man den real existierenden Halsgraben zwischen Vor- und Hauptburg. Genau über diesem Einschnitt sollte, so die Pläne, das Querhaus der Kirche errichtet werden. Ebenso soll jene schmale Felsplatte im Westen, welche für Kreuzgang und Klausurgebäude in der Breite nicht ausreicht, durch ein nördlich angefügtes, außerhalb des Felsens zu erstellendes, auf tiefer liegendem Gelände errichtetes Gebäude ,passend“ gemacht werden, denn nur auf diese Weise ließ sich jene vom Stifter geforderte Idee einer durchgehenden Ost-West-Achse für die gesamte Anlage realisieren.

\section{Die Wasserzuleitung}

Allerdings, was bisher völlig außer Acht gelassen wurde, viel wichtiger noch als ein noch so perfekter Bebauungsplan, ist die Frage: Wie wird das zukünftige Kloster mit dem notwendigen Wasser versorgt? Ohne Wasser kein Kloster!

Sicher war Graf Burkhard als langjähriger Burgherr nicht nur Idealist, Denker und Förderer, sondern genug Realist, um zu wissen, dass die alte Wasserversorgung mittels Ziehbrunnen bzw. Zisternen für das von ihm geplante Kloster unzureichend sein wird. Deshalb war die Schaffung einer leistungsstarken Wasserversorgung noch vor Baubeginn $\mathrm{zu}$ planen und sogar $\mathrm{zu}$ realisieren. Die Quellhorizonte am gegenüberliegenden Hessentaler Hang liegen hoch genug. 


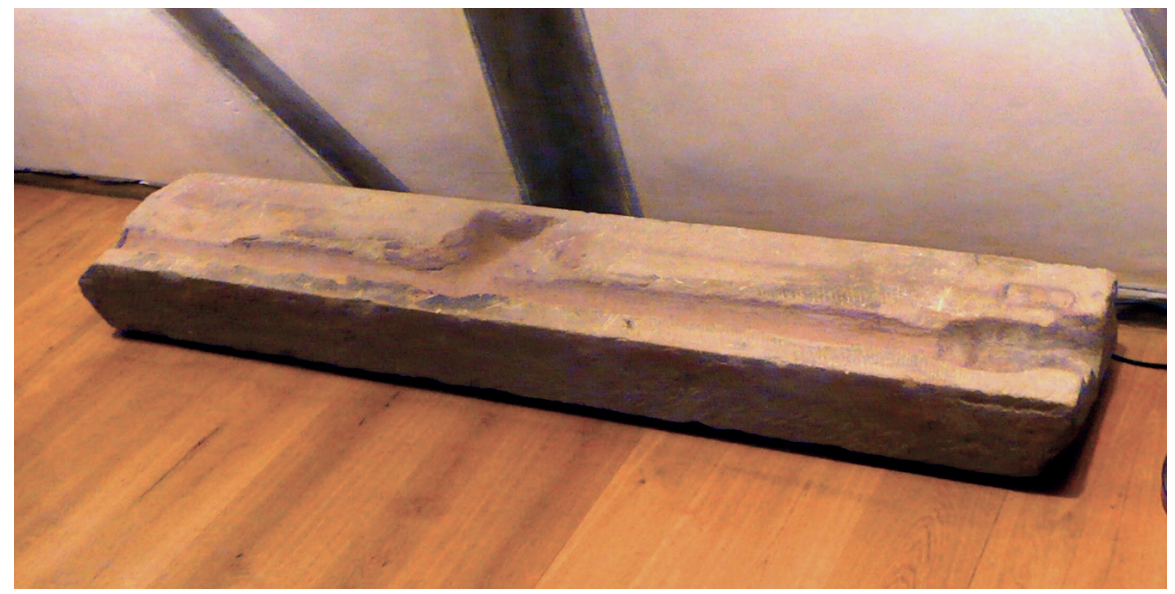

Gussform der Wasserleitung, heute auf der Comburg befindlich (Bild: Christian Schweizer)

Allerdings, um durch das Tal zu kommen, benötigt man eine Leitung aus Röhren, die dem nötigen Druck standhalten. Wer vermochte damals, vor 900 Jahren, ein derartiges, technisch realisierbares Bauwerk zu planen und herzustellen? Offensichtlich lösten Burkhards Spezialisten diese Aufgabe, denn im Untergeschoss des Westturmes soll ein funktionsfähiger Brunnen existent gewesen sein, dessen Abflussrinne 1964 von Eduard Krüger, quer durch den Kreuzgarten führend und in eine dortige Zisterne mündend, freigelegt worden war.

In den 1930er Jahren hatten Mitarbeiter des Denkmalamtes schon einmal nach dem Brunnen im Untergeschoss des Turmes gesucht. Ihren uns vorliegenden Notizen zufolge war aber das Unternehmen erfolglos. Sie stießen auf den ,gewachsenen Fels" und brachen die Arbeiten ab. Dies mag vielleicht der Grund dafür gewesen sein, dass Eduard Krüger in den 40er Jahren bei seinen Grabungen im südlichen Bereich des östlichen Kreuzgangflügels die Suche nach dem Brunnen nicht nochmal aufgenommen hat. Krügers Notizen und Pläne von damals standen uns zur Verfügung.

Nun aber zu jenen uns 1965 gestellten Aufgaben: Wir räumten die in der Barockzeit verfüllte Krypta aus und sammelten dabei viele interessante Erkenntnisse, welche allein einen Vortrag wert wären. Die bedeutendsten Funde aus dem Füllschutt waren jedoch die Reste von farbig gefassten Chorschranken aus Stuck - die ältesten, die wir kennen (jetzt zu sehen im Landesmuseum Württemberg in Stuttgart). Desweiteren konnten wir später an der Südwand des Querhauses, also jetzt in der Wintersakristei, die Gestaltung der Außenfassaden der romanischen Kirche erkunden und dort sichtbar belassen. Außerdem konnten wir den nördlichen Bereich des östlichen Kreuzgangflügels untersuchen und damit Krügers Befunde von 1940/42 aus dem gegenüberliegenden, südlichen Bereich bestätigen. 


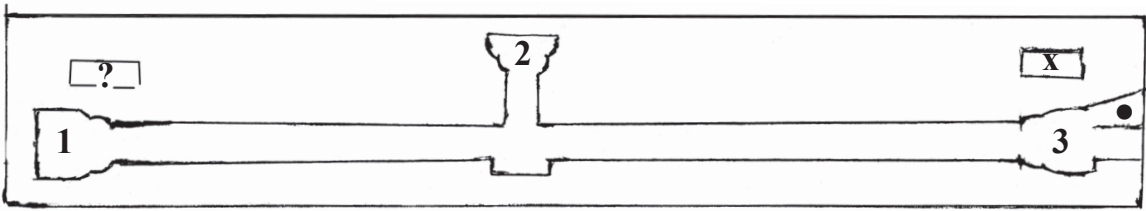

Zeichnung der Gussform der Wasserleitung auf der Comburg:

Eine Hälfte der Gussform mit Formschloss $x$.

1: Endmuffe, 2: Abzweigmuffe, 3: Obere Muffe mit Einfüllöffnung (Bild: Rolf Schweizer)

Der östliche Kreuzgangflügel verlief über Stufen tiefer gelegt unter dem westlichen Hauptaltar bzw. dessen Aufgang hindurch, und man konnte über Stufen von unten aus in die geistlichen Räume des westlichen Kirchenbereichs gehen. An der tiefsten Stelle lag der Zugang ins Brunnenhaus im Untergeschoss des Turmes. Hatte diese Tieferlegung des Brunnens und des östlichen Kreuzgangflügels vor 900 Jahren vielleicht sogar seinen Grund in den Druckverhältnissen der Wasserversorgung? Das kann möglicherweise durch ingenieurmäßige Untersuchungen geklärt werden.

Mit seinen abschnittsweisen Tieferlegungen ist in seiner Machart dieser östliche Kreuzgangflügel schon allein ein bauliches Unikum, aber er wurde allerdings noch übertroffen durch jene beiden nach Süden und nach Norden weiterführenden Fortsätze. Nach Süden wurde dadurch das Ostende des großen Vikarienbaues, der Kapitelsaal, erschlossen, und nach Norden erreichte damit der Gang eine Kapelle am östlichen Abschluss, des sog. Adelmannbaus. Dort ist das große Ostfenster dieser Kapelle noch vorhanden. Der ehemalige, um vier Stufen tiefer liegende Raum der Johanneskapelle dient jetzt als Treppenhaus. Seine Säulengalerie, wie jene im Kapitelsaal, existierte ehedem auch hier. Die Symmetrie der Kreuzgang-Anlage ist bis auf diesen bedauerlichen barockzeitlichen Eingriff bis heute existent.

Nach all diesen Erkenntnissen fehlte nur noch der Brunnen, und wir hegten Zweifel an den Angaben unserer Vorgänger aus den 1930er Jahren. Allerdings erkannten wir jenen 1930 vermeintlich erschlossenen, „anstehenden“ Fels als ortsfremden Keupersandstein, also nicht als den tatsächlich anstehenden $\mathrm{Mu}$ schelkalkfels, auf dem das Turmfundament ruht.

\section{Der Brunnen}

Wir legten den Sandsteinblock frei, der sich dann als unterstes Brunnenbecken zu erkennen gab. Dieses schalenartig bearbeitete runde Werkstück kann seiner Größe wegen nur zeitgleich mit dem Fundament des Turmes eingebaut worden sein. Im Zentrum des Bodenbeckens scheint ein Brunnenstock gestanden zu ha- 


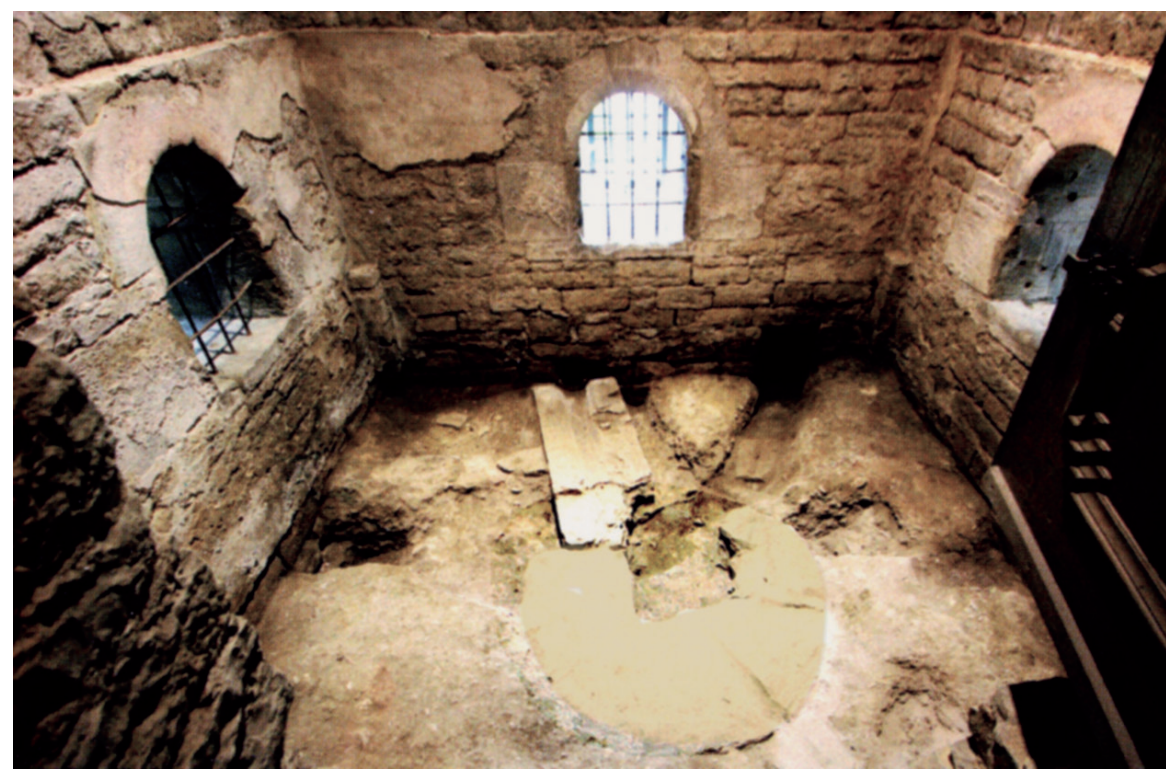

Brunnenkapelle (Bild: Michael Greiner [Red.]:

Die Comburgen bei Schwäbisch Hall. Stuttgart 2010)

ben und eine Abflussrinne führt nach Westen hinaus in den Kreuzgarten. Von Nordosten her erfolgte die Wasserzufuhr ehedem über Leitungsrohre, deren Trasse mit rinnenartig ausgehöhlten Lagersteinen sichtbar wurde. Offensichtlich führte vom Brunnen aus eine abzweigende Leitung nach Nordwesten, hinüber zur Küche bzw. zur Abtei. Die Frage, die sich nun stellte, war: Wie mögen die Rohre dieser Druckleitung vor 900 Jahren ausgesehen haben und aus welchem Material waren diese wie gestaltet? Eine Wasserleitung von mehr als einem Kilometer Länge, die durch das Tal auf den Berg zu führen war, ist für die damalige Zeit ein technisches Meister- und Wunderwerk. Ihre Rohre aus Ton zu fertigen und zu brennen oder sie als hölzerne Deichelleitung zu bauen, war wegen des Druckes, der auf dieser Leitung lag, wohl undenkbar. Völlig utopisch aber schien uns nach unserem damaligen Kenntnisstand eine Leitung aus bronzenen Röhren. So etwas gab es doch seinerzeit noch gar nicht - ganz zu schweigen von den Kosten des Metalls!

Spätestens in barocker Zeit wurde unsere Leitung abgebaut. Mit dem Bau der neuen Kirche wurde auch der Brunnen im Untergeschoss des Westturmes aufgegeben. Könnten dort aber noch irgendwelche Reste von den Röhren zu finden sein? Beim aufmerksamen Untersuchen der Abbruchschicht konnten drei Objekte als Hinweis auf die verlorene Leitung gefunden werden: Erstens ein etwa daumengroßes Bruchstück von der Seitenwand einer bronzenen Röhre mit ca. 7-10 mm Wandstärke; dann ein in Schichten abgelagertes Stück Kalksinter, ca. 
$50 \mathrm{~mm}$, das aus einer solchen Röhre stammen und uns Auskunft geben könnte über den inneren Durchmesser der Leitungsrohre. Drittens kamen im Abstand von jeweils ca. 1,8 m drei Reste von verklopften Bleischnüren zutage, welche mit der Technik des Abdichtens der Anschlussstellen in Verbindung zu bringen sind. Von jetzt ab waren wir uns bewusst: Die Komburg birgt die Reste einer technisch einzigartigen Versorgungsanlage, nämlich einer Druckleitung aus Bronze und das aus der Zeit vor 900 Jahren. Gibt es Beispiele dafür anderen Ortes? Wer konnte dafür die notwendigen Kenntnisse haben und wie sollten die Rohre aus Metall dafür gefertigt worden sein? Fragen über Fragen! Die einschlägigen Handbücher zur Wasserversorgung im Allgemeinen und zu Wasserleitungen im Besonderen während des Mittelalters und der Frühen Neuzeit befassen sich durchweg mit späteren Zeiten und nicht mit dem 11. Jahrhundert. ${ }^{4}$ Es gibt aber eine lange nach unserer Grabung erschienene, exzellente Einzelstudie aus dem Jahr 1991, die sich intensiv mit der Wasserversorgung im Mittelalter im Allgemeinen und in Klöstern im Besonderen beschäftigt. ${ }^{5}$

Dann rief man uns anfangs der 1970er Jahre nochmals in den Bereich des Ostchores. Dort beabsichtigte das Bauamt, für die ausgelagerten sterblichen Reste der beiden Dekane, zunächst des Dekans Ulrich zu Guttenberg (als seinerzeitigem Bauherren) und dann des Dekans zu Erthal, neue Grüfte anzulegen, wozu ein Platz westlich der Krypta und nördlich der Mittelachse vorgesehen war. Wie zu erwarten, konnten auch dort im Füllschutt weitere Chorschrankenfragmente gefunden werden, und in der Mittelachse entdeckten wir eines der alten Stiftergräber. Die Grabstätte lag, verfüllt mit barockem Abbruchschutt, ohne Abdeckplatte vor uns.

4 Günter Bayerl: Historische Wasserversorgung. Bemerkungen zum Verhältnis von Technik, Mensch und Gesellschaft. In: Ulrich Troitzsch / Gabriele Wohlauf(Hg.): Technikgeschichte. Historische Beiträge und neuere Ansätze (Suhrkamp Taschenbuch Wissenschaft 319). Frankfurt/M. 1980, S. 180 221; Bayerl nennt als älteste Leitungen solche aus dem 15. Jahrhundert. Das gleichzeitig erschienene, seinerzeit vieldiskutierte Werk von Jean Gimpel: Die industrielle Revolution des Mittelalters. Zürich, München 1980, S. 39 ff und $44 \mathrm{ff}$ nennt zwar den seit dem 11. Jahrhundert aufblühenden Metallbergbau, geht aber auf Spezialprobleme wie eventuelle frühe Wasserleitungen aber nicht ein. - Auch ansonsten wird, was mittelalterliche Metallverarbeitung angeht, in den Handbüchern und Überblickswerken das 11. Jahrhundert weitgehend ausgeblendet. Der Schwerpunkt liegt auf späteren Jahrhunderten, vgl. die von verschiedenen Autoren verfassten Metallkapitel bei Uta Lindgren (Hg.): Europäische Technik im Mittelalter. 800 bis 1400. Tradition und Innovation. Berlin 1996, S. 137-220; und das Metallkapitel bei Günter Bayerl: Technik in Mittelalter und Früher Neuzeit. Darmstadt 2013, S. 135-162; Sven Hauschke: Das Metallhandwerk. Eisen-, zinn- und kupferverarbeitende Gewerbe. In: Christine Sauer (Hg.): Handwerk im Mittelalter. Darmstadt 2012, S. 57-70. Die Archäometallurgie hat dagegen ihren Schwerpunkt auf weiter zurückliegenden Epochen und beschäftigt sich jedenfalls nicht mit bronzenen Wasserleitungen, vgl. Paul D. Craddock: Early Metal Mining and Production. Edinburgh 1995; Christian Strahm: Helvetia Archaeologica 25 (1997), S. 2-39.

5 Frontinus-Gesellschaft (Hg.): Die Wasserversorgung im Mittelalter (Geschichte der Wasserversorgung 4). Mainz 1991. Zu Klöstern insbesondere, knapp: Klaus Grewe: Wasserversorgung und -entsorgung im Mittelalter. In: ebd., S. 11-88, hier insbesondere 32-48; zu Klöstern ausführlich: Clemens Kosch: Wasserbaueinrichtungen in hochmittelalterlichen Konventanlagen Mitteleuropas. In: ebd., S. 89-148; vgl. auch: C. James Bond: Mittelalterliche Wasserversorgung in England und Wales. In: ebd., S. 149-184; Paul Benoit / Monique Wabont: Mittelalterliche Wasserversorgung in Frankreich. Eine Fallstudie: Die Zisterzienser. In: ebd., S. 185-228. 


\section{Das Stiftergrab im Ostchor und sein Bezug zur Wasserleitung}

Ein steinerner Sarg im Boden war aus plattenartigen Sandsteinen zusammengesetzt. Sorgfältigst wurde der Füllschutt nach Spuren eines des darin begrabenen Stifters durchsucht. Um 1220/30 sind, wie bekannt, die Gebeine der vier Stifterpersönlichkeiten aus ihren Grabstätten geborgen und, je nach Person geordnet, in die bekannte steinerne Tumba übertragen worden. Die uns gestellte Frage lautete: Welcher der vier Männer lag hier und können wir dies nach 900 Jahren noch klären? Wir konnten es noch! Im steinernen Sarg fanden sich kleine Skelett-Teile, welche sich einwandfrei dem Skelett des Grafen Burkhard zuordnen ließen, so z.B. fehlte der gefundene verknöcherte Kehlknorpel nur bei seinem Skelett. Also hatten wir das Grab des eigentlichen Stifters gefunden, und in der Folge wurde später die Tumba im Chor genau über dieser Grablege aufgestellt. Der Graf starb als Mitglied des Klosterkonvents, also als einfacher Mönch, im Jahr 1096/97.

Vielmehr allerdings als nur das Grab des Stifters wurde die Grabstätte selbst zu einem historisch, technischen, archäologischen Kleinod, denn als südliche Seitenwand des steinernen Sarges - also rechtsseitig entlang des darin ehedem Bestatteten - war ganz gezielt ein spezieller Stein eingebaut, der meines Erachtens explizit dafür gedacht war, der Nachwelt die Genialität des Stifters zu vermitteln. Der Stein sollte zeigen, welch außergewöhnlicher Mensch hier begraben lag. Was erzählt uns dieses Werkstück? Es berichtet uns vom Fortschritt der handwerklichen Kunst und Technik damaliger Zeit. Der Stein war als Metallgussform perfekt zugerichtet worden und bildete zusammen mit einer zweiten spiegelbildlich gearbeiteten, jedoch verlorenen Hälfte eine Gussform für etwa 2 m lange Bronzeröhren. ${ }^{6}$ Die vorliegende Form wurde nach mehrfachem Ge-

6 Zur Wasserleitung auf der Komburg allgemein: Clemens Kosch: Klausurquadrum, Westchorturm und Brunnenstube der Großcomburg. Ein Beitrag zur Erforschung axialer Konventsanlagen des Hochmittelalters. In: WFr 65 (1981), S. 5-50, insbesondere S. 48 f die Fotos und Lagepläne von Brunnen und Wasserleitung; ders.: Die Wasserleitung vom Ende des 11. Jahrhunderts im ehemaligen Kloster Großcomburg. In: Frontinus-Gesellschaft (wie Anm. 5), S. 237-243 (mit zahlreichen Abbildungen und Plänen). Kosch geht davon aus, dass die Wasserleitung einen Laufbrunnen speiste. Sie muss - nach dem Prinzip der kommunizierenden Röhren - von einer weit außerhalb des Klosters liegenden Quelle gespeist worden sein, die höher lag als ihr Brunnenbecken auf der Komburg. Kosch geht - anders als die Ausgräber - davon aus, dass es sich bei der Platte im Grab Burkhards nicht um eine Gussform handelt, weil die Gusskanäle fehlen. Vielmehr handle es sich bei der Platte um eine „Umhüllung der Wasserleitung“, die dann aber nicht wie vorgesehen zum Einsatz kam, sondern „,ausgesondert worden“ sei. Die Deutung Koschs erklärt indessen nicht die hitzebedingte rote und schwarze Verfärbung der Platte. Gusskanäle könnten in der anderen - nicht erhaltenen Gegengussplatte vorhanden gewesen sein. - Kosch verweist ferner auf die zwischenzeitlich ausgegrabene Wasserleitung des Klosters Hirsau, die große Ähnlichkeiten mit der Wasserleitung den Komburg aufweist. Er vermutet in Hirsau sogar denselben Mäzen wie auf der Komburg, nämlich der Mainzer Bürger Wignand von Kastell, der als Mönch auf der Komburg starb und zusammen mit Graf Burkhard, dessen Bruder Graf Heinrich, dem ersten Abt, in der Stiftertumba begraben wurde. Vgl. zu Hirsau: Otto Teschauer: Archäologische Beobachtungen zur Wasserversorgung des Klosters Hirsau im Mittelalter. In: Frontinus-Gesellschaft (wie Anm. 5), S. 244-257. 
brauch ausgemustert. Die Spuren der Gießvorgänge sind als schwarze Rußflächen genauso zu erkennen, wie auch der Stein sich von der Hitze des Metalls rot verfärbt hat. Letztendlich sind nach mehreren Güssen die scharfen Steinkanten abgesplittert und damit war die Form unbrauchbar geworden. Das vorliegende Werkstück diente allerdings nicht zum Guss nur einfacher Röhren, sondern aus ihm wurden solche mit zusätzlichen Abzweigmuffen oder sogar mit Abstellhahn gefertigt. Alle diese Röhren endeten mit einer Steckmuffe und wurden wohl nach dem Zusammenfügen mit Hanf bzw. Werg abgedichtet und danach mit Bleikordeln verklopft. ${ }^{7}$ Das gefundene Werkstück gilt als einzigartig und ordnet sich, was die Kunstfertigkeit, handwerkliches Können und den technischen Fortschritt auf der Komburg anbetrifft, mit in die vielfältigen Meisterleistungen ein, wie Chorschranken, Malerei (Wand und Buch), Metall (Antependien und Kronleuchter), Orgelbau und nun auch Metallguss. Unser Objekt ist der Beleg dafür, dass all dies vor Ort, auf der und um die Komburg, vor 900 Jahren geleistet worden ist.

Letztlich ist es den Ausgräbern und Archäologen vor 50 Jahren mehr als schwer gefallen, diese einzigartige Grabstätte Burkhards zu „plündern“. Die Gussform, die zweifellos als besondere Grabbeigabe gedacht war, wurde, ihrer Bedeutung wegen, aus dem Grab genommen, um sie der Forschung anschließend zur Verfügung zu stellen. Es war gewiss nicht gedacht, dieses kulturgeschichtlich, technische Werkstück vergessen und völlig falsch deklariert auf der Komburg zum „Dauerschlaf" einzumotten.

Diese Form gehört in die Hand einer Forschungsstätte und die Wasserleitung und Brunnentechnik der Komburg gehört in die Hände zuständiger Ingenieure z. B. (Stadtwerke Hall) Wasser- und Leitungsbautechnik. Meinen Vortrag möchte ich allerdings nicht beenden, ohne diese müßige Frage zu stellen: Hat der Stiftergraf seine Grabstätte schon vor oder mit der Weihe der Kirche 1088, also zu Lebzeiten, anlegen lassen, was nicht abwegig wäre? Und demnach hätte er gezielt auf den Einbau der Gussform Wert gelegt, um damit zu zeigen, dass hier ein Zentrum von Wissenschaft und Kunst entstehen wird. Gerade dieser Stein könnte als Indiz dafür gedeutet werden. Den Stifter möchte ich, 920 Jahre nach

7 Kosch 1981 (wie Anm. 6) und ders. 1991 (wie Anm. 5 bzw. 6) stellt die Deutung der Gussform als solche in Abrede und gibt dafür den Hinweis auf jene gefundenen Leitungs-Lagersteine, mit welchen er die Gussform gleichsetzt. Diese in situ gefundenen Lagersteine sind aber rechteckige Quader mit grob ausgehauener Mittellängsrinne, in welche die Röhren meines Erachtens sorgsam auf Sand bzw. Lehm gelegt waren. Dass es sich bei der gefundenen Formhälfte tatsächlich aber um eine diffizil gearbeitete Gießform handelt, zeigt sich jedoch deutlich an der Anbringung von zwei sog. „Formschlössern“, die ein Verschieben der beiden Formteile verhindert haben. Außerdem ist unsere Gussformhälfte oben, unten und wegen des eingebauten Abzweigstutzens auch seitlich mit abgeschlossenem Muffen-Ende als Schlussplatte versehen. Der Hinweis von Kosch auf fehlende Luftkanäle ist hinfällig, da die beiden Formhälften auf die Länge sicher nicht miteinander genau geschlossen haben. Sie wurden zum Guss mit Lehm z. T. abgedichtet. Zum Schluss besitzt die Form noch einen am oberen Ende angebrachten Einguss-Stutzen, den Kosch gar nicht erwähnt. Die Technik des Gießens von dünnwandigen Röhren mit verlorenem Innenkern mag von zuständigen Spezialisten zu lösen sein. 
seinem Tod, als genialen Zeitgenossen bezeichnen. Die Mannschaft, die ihn umgab, bildete noch weitere 150 Jahre lang einen Kondensationspunkt von Kunst und Können.

Anschließend an das Referat fand eine Begehung des Bereichs östlicher Kreuzgang - Kapitelsaal - Kirche - Adelmannbau mit Blick in den Brunnen statt, bevor es - zurück in der Kirche - zur Wintersakristei, Krypta mit dem Ort der früheren Grablege Guttenberg/Erthal und dann zur Tumba und zur neuen Grablege Guttenberg/Erthal ging. Den Abschluss bildete der Gang zur neuen Dekanei mit dem Löwen und der Physiologus-Geschichte, der als Seelmessenkapelle gedeuteten Sechseck-Kapelle und zuletzt zur Gussform der Wasserleitung in der alten Dekanei. 